\title{
A state-space modeling approach for active structural acoustic control ${ }^{1}$
}

\author{
Leopoldo P.R. de Oliveira ${ }^{\mathrm{a}, \mathrm{b}}$, Paulo S. Varoto ${ }^{\mathrm{a}, *}$, Paul Sas $^{\mathrm{b}}$ and Wim Desmet $^{\mathrm{b}}$ \\ ${ }^{a}$ University of São Paulo, Engineering School of São Carlos, Dynamics Lab. Av. Trabalhador Sancarlense, 400, \\ 13566-590 São Carlos-SP, Brasil \\ ${ }^{\mathrm{b}}$ Katholieke Universiteit Leuven, Department of Mechanical Engineering, Celestijnenlaan 300B, 3000 Leuven, \\ Belgium
}

Received 27 March 2008

Revised December 2008

\begin{abstract}
The demands for improvement in sound quality and reduction of noise generated by vehicles are constantly increasing, as well as the penalties for space and weight of the control solutions. A promising approach to cope with this challenge is the use of active structural-acoustic control. Usually, the low frequency noise is transmitted into the vehicle's cabin through structural paths, which raises the necessity of dealing with vibro-acoustic models. This kind of models should allow the inclusion of sensors and actuators models, if accurate performance indexes are to be accessed. The challenge thus resides in deriving reasonable sized models that integrate structural, acoustic, electrical components and the controller algorithm. The advantages of adequate active control simulation strategies relies on the cost and time reduction in the development phase. Therefore, the aim of this paper is to present a methodology for simulating vibro-acoustic systems including this coupled model in a closed loop control simulation framework that also takes into account the interaction between the system and the control sensors/actuators. It is shown that neglecting the sensor/actuator dynamics can lead to inaccurate performance predictions.
\end{abstract}

Keywords: Vibro-acoustics, active-control, state-space

\section{Nomenclature}

$\mathbf{A}=$ state-space dynamic matrix

$\mathbf{B}=$ state-space input matrix

$[b]=$ matrix composed by zeros and ones for state-space formulation

$[c]=$ matrix composed by zeros and ones for state-space formulation

$\mathbf{C}=$ state-space output matrix

D $=$ damping matrix

$d=$ lumped damping

$\mathbf{F}=$ load vector

$\mathbf{I}=$ identity matrix

$I=$ current

$j \quad=$ imaginary number

$\mathbf{K}=$ stiffness matrix

$k=$ lumped stiffness

\footnotetext{
${ }^{1}$ Based on a paper presented at the XII International Symposium on Dynamic Problems of Mechanics (DINAME 2007), Ilhabela, SP, Brazil, February 26 - March 2, 2007.

${ }^{*}$ Corresponding author: EESC-USP-Mechanical Engineering Department, Av. Trabalhador Saocarlense 400, 13566560 Sao Carlos-SP Brazil. Tel.: +55 16 33739423; Fax: +16 16 33739402; E-mail: varoto@sc.usp.br.
} 


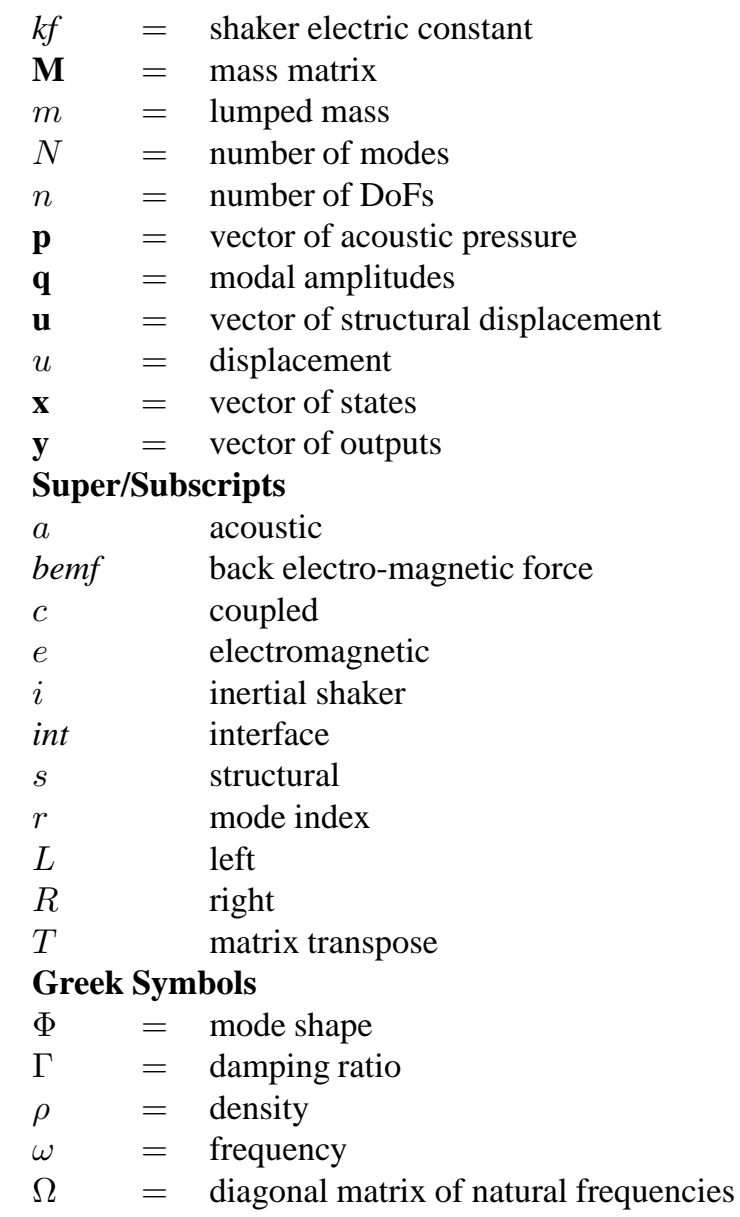

\section{Introduction}

The demands for improvement in sound quality and reduction of noise generated by vehicles are constantly increasing, as well as the penalties for space and weight of the passive control solutions. A promising approach to cope with this challenge is the use of active structural-acoustic control (ASAC). During the design phase, simulation plays an important role in predicting the performance and feasibility of active control solutions. As a result, the demands for more reliable simulation techniques are also ever-increasing. The advantages of adequate simulation strategies rely on the time and cost reduction in the development phase, enabling the engineer to try different schemes, sensors and actuators configuration and control strategies with minimum physical prototyping.

Usually, the low frequency noise is transmitted into the vehicle cabin through structural paths, which raises the necessity of dealing with vibro-acoustic models. This kind of models allows the use of acoustic disturbance and secondary sources. It should also allow the inclusion of sensors and actuators models, not only in the sense that their own dynamics may significantly change the original system dynamics, but also that they can pose frequency, phase or amplitude limitations to the control performance. The challenge thus resides in deriving reasonable sized models that integrate acoustic, structural and electrical components together with the controller algorithm.

Therefore, the aim of this paper is to present a methodology for simulating vibro-acoustic systems, including this coupled model in a closed loop control simulation framework that also takes into account the interaction between the system and the control actuators and sensors. This methodology consists of deriving a fully coupled finite element (FE) model of the vibro-acoustic system which is reduced and formulated as a modal state-space model into Matlab/Simulink, where, eventually, models for sensors and actuators are included and the controller implemented. 


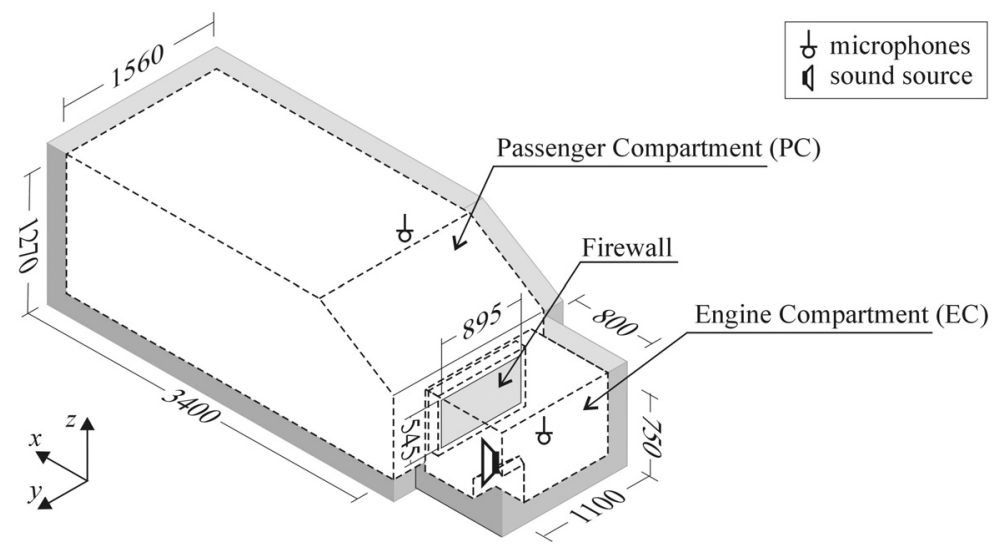

Fig. 1. Schematic view of the system under study (dimensions in $\mathrm{mm}$ ).

To demonstrate the proposed modeling procedure, a vehicle mock-up is selected (Fig. 1). It consists of a simplified car cavity with rigid walls, to provide well-defined acoustic boundary conditions, thus avoiding uncertainties during the vibro-acoustic modeling phase. A sound source placed in the engine compartment (EC) works as a primary disturbance source. A flexible firewall allows the noise generated in the engine compartment (EC) to be transmitted to the passenger compartment (PC). Collocated velocity feedback is selected as control strategy, due to its relatively simple implementation and guarantied stability. Decentralized collocated sensor/actuator pairs (SAPs) are placed on the firewall in order to reduce the noise transmitted from the EC to the PC.

The modeling procedure for the fully coupled vibro-acoustic system, the modal state-space formulation and the SAP models are presented in Section 2. The numerical results for the passive and the selected active systems are treated in Section 3. Finally, some conclusions are addressed in Section 4.

\section{Modeling procedure}

Bringing research results on intelligent materials to the level of industrial applications requires the design processes of active systems to become part of the complete product development cycle. In other words, it is necessary to extend the use of simulation models, which are the cornerstone of today's design process, to enable the integration of advanced materials, active systems, actuators, sensors and control algorithms. Moreover, it must be possible to integrate these models into system level virtual prototype models [1]. It is clear that no single integrated solution will be able to fulfill all requirements of the various material and control approaches; therefore the focus of this research is on supporting, as much as possible, the use, combination and extension of existing codes and tools.

Considering a closed compartment, any airborne noise generated outside this cavity can only be perceived by the occupants if transmitted through structural paths. That is the case of the engine noise generated in the EC, which is transmitted to the PC via the firewall. This fluid-structure interaction imposes the necessity of dealing with fully coupled vibro-acoustic models, which are usually computationally expensive. An advantage though, is that fully coupled FE models allow the use of simultaneous acoustic and structural inputs and outputs, which, from the controller perspective, means being able to include either structural or acoustic disturbance, secondary sources and sensors of either kind. Another advantage of using fully-coupled vibro-acoustic models is the accuracy of the estimated control performance, as an uncoupled analysis can overestimate the controller efficiency [2].

Therefore, the first challenges in ASAC simulation resides in deriving reasonable sized vibro-acoustic models which can be integrated in the control simulation environment. This can be accomplished by deriving a fully coupled FE model of the vibro-acoustic system, which is reduced and formulated as a state-space model into Matlab/Simulink where the controller is implemented (Fig. 2).

The models for sensors and actuators can be included directly in the control design environment. If done in this phase, any eventual changes in the SAPs (positions, specifications or even kind) will not demand a recalculation of 
Table 1

Component model type and software

$\begin{array}{ccc}\text { component } & \text { model type } & \text { Software } \\ \text { Cavities } & \text { FEM } & \text { LMS.Sysnoise } \\ \text { Firewall } & \text { FEM } & \text { Patran / Nastran } \\ \text { inertial shaker } & \text { State-Space } & \text { Matlab / Simulink } \\ \text { controller } & \text { State-Space } & \text { Matlab / Simulink }\end{array}$

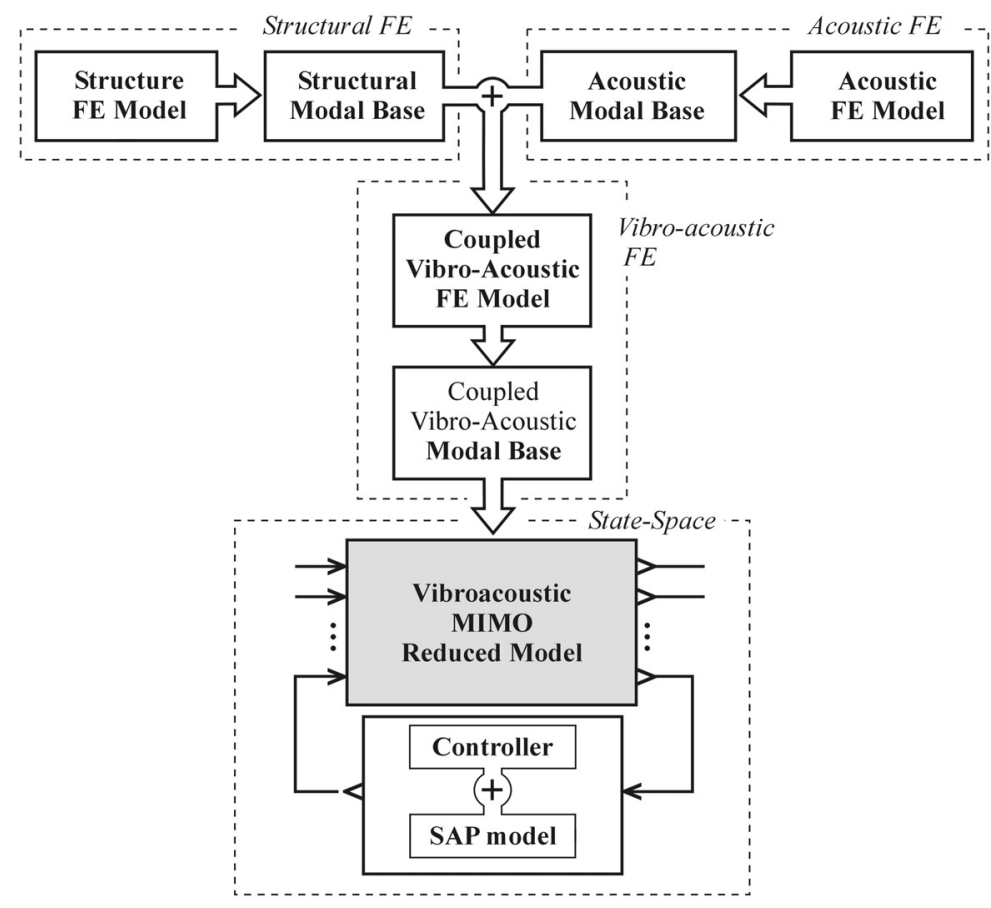

Fig. 2. Modeling procedure scheme.

the full vibro-acoustic system. The inclusion of sensor and actuator models contributes for the model accuracy, not only in the sense that their own dynamics may significantly change the original system response, but also that they can present frequency, phase or amplitude limitations to the control performance.

The full modeling procedure for the present case study is illustrated in Fig. 2. It starts with uncoupled structural and acoustic FE models for the structure and the cavities. An uncoupled modal base is extracted for both models. The vibro-acoustic FE model consists of a modal model with a coupled modal base built with the uncoupled modal bases. The vibro-acoustic modal base is then used to derive a state-space model, which can be used in the control design environment. The different model types and software used for each component are show in Table 1.

If the sensor and/or actuator electro-mechanical behavior is considered to be negligible, this modal state-space model allows the implementation of control systems with idealized inputs and outputs, namely structural force and volume velocity excitation. Some preliminary results can be achieved with such models, although an accurate access of the controller performance requires a more comprehensive model of the SAP. The next sub-sections describe in detail each one of the required steps.

\subsection{Vibro-acoustic modeling}

One of the coupled vibro-acoustic FE/FE formulation is the Eulerian, in which the structural degrees of freedom (DoFs) are displacement vectors, while the acoustic DoFs are expressed as scalar functions. The latter is usually the 


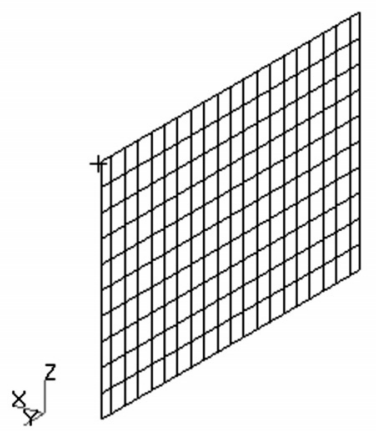

(a)

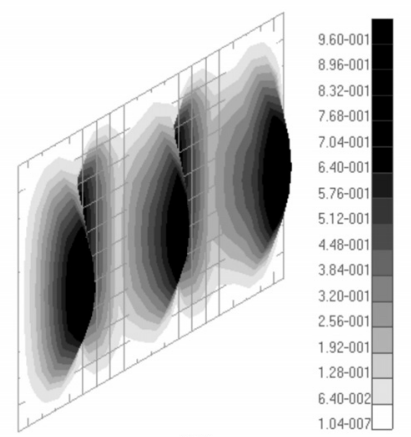

(b)

Fig. 3. Firewall: (a) FE mesh and (b) structural mode at $160 \mathrm{~Hz}[5 ; 1]$.

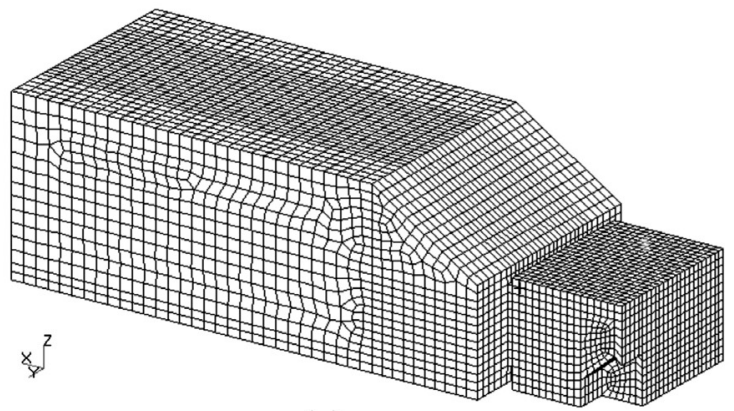

(a)

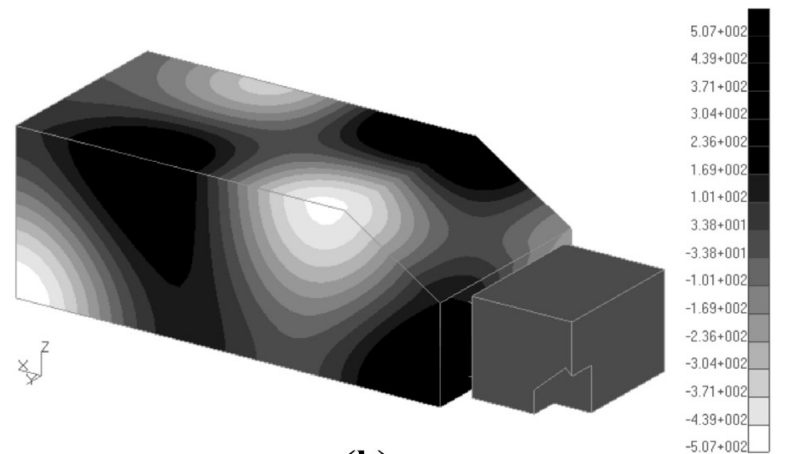

(b)

Fig. 4. Mock-up cavities: (a) FE mesh and (b) uncoupled mode at $192.5 \mathrm{~Hz}$.

acoustic pressure, which yields non-symmetrical mass and stiffness matrices, posing a disadvantage to FE solvers [3, 4].

The vibro-acoustic FE modeling of vehicle interiors always requires the setup of FE meshes for both, vehicle structure and interior cavities. Since the acoustic wavelengths are usually longer than the structural ones in the low frequency range, an optimized acoustic mesh can be much coarser than the structural mesh. However, if the meshes are compatible, some intermediate numerical steps can be neglected resulting in a simplified procedure [5]. Therefore a trade-off choice for the size of structural and acoustic FE meshes was taken. In this way, they present coinciding nodes without affecting the accuracy of the predicted results within the frequency range of interest ( 0 to $200 \mathrm{~Hz}$ ).

The structural mesh is shown in Fig. 3(a) with a total of 231 nodes and 200 4-noded shell elements. The steel firewall, $1.5 \mathrm{~mm}$ tick, presents 13 modes from 0 to $200 \mathrm{~Hz}$. The structural mode shape presenting the shortest wavelength is the [5;1] well described by this number of elements (Fig. 3b).

The element type chosen for the acoustic mesh is the 8-noded brick (hexahedral), not only by the smaller number of elements needed, but also due to its higher accuracy in post processing pressure derived quantities (velocity and sound intensity) when compared to tetrahedral elements [6]. The maximum frequency of interest (200 $\mathrm{Hz}$ for this case study) was also taken into account, so that the acoustic model could have the minimum number of elements. The resulting mesh (with 26050 elements and 23196 acoustic DoFs) and the highest mode shape in the frequency band are depicted in Fig. 4. With respect to the elements size, this acoustic model is valid until $514 \mathrm{~Hz}$, considering 6 elements per wavelength, which is fairly suitable for this application.

As mentioned before, the structural and acoustic models are fully-coupled in this FE/FE modeling approach. The effect of the interfacing fluid on the structure dynamics can be considered as a pressure load on the wet surface, thus turning the structural differential equation into the form of Eq. (1). 


$$
\left(\mathbf{K}_{s}+j \omega \mathbf{D}_{s}-\omega^{2} \mathbf{M}_{s}\right) \mathbf{u}(\omega)+\mathbf{K}_{c} \mathbf{p}(\omega)=\mathbf{F}_{s}(\omega)
$$

where $\mathbf{K}_{s}, \mathbf{D}_{s}$ and $\mathbf{M}_{s}$ are respectively the structural stiffness, damping and mass matrices, $\mathbf{K}_{c}$ is the coupling matrix, $\mathbf{u}$ is the vector of structural displacements, $\mathbf{p}$ is the vector of acoustic pressures and $\mathbf{F}{ }_{s}$ is the structural load vector.

In a similar way, the structural vibration works as an extra acoustic input and therefore must be taken into account as:

$$
\left(\mathbf{K}_{a}+j \omega \mathbf{D}_{a}-\omega^{2} \mathbf{M}_{a}\right) \mathbf{p}(\omega)-\omega^{2} \mathbf{M}_{c} \mathbf{u}(\omega)=\mathbf{F}_{a}(\omega)
$$

where $\mathbf{K}_{a}, \mathbf{D}_{a}$ and $\mathbf{M}_{a}$ are the acoustical stiffness, damping and mass matrices, $\mathbf{M}_{c}$ is the coupling matrix, and $\mathbf{F}_{a}$ is the acoustic load vector. For the sake of brevity any function ' $h(\omega)$ ' is represented just as ' $h$ ' hereafter.

Regarding the special relation, $\mathbf{K}_{c}=-\rho_{0} \mathbf{M}_{c}^{T}[7,8]$, the combined system of equations in this Eulerian FE/FE formulation yields:

$$
\left(\left[\begin{array}{cc}
\mathbf{K}_{s} & \mathbf{K}_{c} \\
\mathbf{0} & \mathbf{K}_{a}
\end{array}\right]+j \omega\left[\begin{array}{cc}
\mathbf{D}_{s} & \mathbf{0} \\
\mathbf{0} & \mathbf{D}_{a}
\end{array}\right]-\omega^{2}\left[\begin{array}{cc}
\mathbf{M}_{s} & \mathbf{0} \\
-\rho_{a} \mathbf{K}_{c}^{T} & \mathbf{M}_{a}
\end{array}\right]\right)\left\{\begin{array}{l}
\mathbf{u} \\
\mathbf{p}
\end{array}\right\}=\left\{\begin{array}{l}
\mathbf{F}_{s} \\
\mathbf{F}_{a}
\end{array}\right\}
$$

Based on Eq. (3) it is clear that the resulting vibro-acoustic system is coupled, though it is no longer symmetric. As a consequence of this non-symmetric nature, the solution of the associated undamped eigenproblem is computationally more demanding and results in different left and right eigenvectors:

$$
\begin{aligned}
& {\left[\begin{array}{cc}
\mathbf{K}_{s} & \mathbf{K}_{c} \\
\mathbf{0} & \mathbf{K}_{a}
\end{array}\right]\left\{\Phi_{R}\right\}_{r}=\omega_{r}^{2}\left[\begin{array}{cc}
\mathbf{M}_{s} & \mathbf{0} \\
-\rho_{a} \mathbf{K}_{c}^{T} & \mathbf{M}_{a}
\end{array}\right]\left\{\Phi_{R}\right\}_{r}, \quad r=1,2, \ldots,\left(n_{a}+n_{s}\right)} \\
& \left\{\Phi_{L}\right\}_{r}^{T}\left[\begin{array}{cc}
\mathbf{K}_{s} & \mathbf{K}_{c} \\
\mathbf{0} & \mathbf{K}_{a}
\end{array}\right]=\omega_{r}^{2}\left\{\Phi_{L}\right\}_{r}^{T}\left[\begin{array}{cc}
\mathbf{M}_{s} & \mathbf{0} \\
-\rho_{a} \mathbf{K}_{c}^{T} & \mathbf{M}_{a}
\end{array}\right], \quad r=1,2, \ldots,\left(n_{a}+n_{s}\right)
\end{aligned}
$$

where $r$ is the index of the coupled natural frequency $\omega_{r}, \Phi_{R}$ and $\Phi_{L}$ are, respectively, the left and right coupled modes, $n_{a}$ and $n_{s}$ are the number of retained acoustic and structural modes, respectively.

Moreover, it has been indicated [8] that, for the Eulerian formulation, the left and right eigenvectors can be related as:

$$
\left\{\Phi_{L}\right\}_{r}=\left\{\begin{array}{c}
\left\{\Phi_{L s}\right\}_{r} \\
\left\{\Phi_{L a}\right\}_{r}
\end{array}\right\}=\left\{\begin{array}{c}
\left\{\Phi_{R s}\right\}_{r} \omega_{r}^{2} \\
\left\{\Phi_{R a}\right\}_{r}
\end{array}\right\}, \quad r=1,2, \ldots,\left(n_{a}+n_{s}\right)
$$

A common practice in solving such vibro-acoustic problems is the use of component mode synthesis (CMS). It consists of expanding the structural DoFs in terms of a set of $N_{s}$ uncoupled structural modes $\Phi_{s}$ (without any acoustic pressure load along the coupling interface), as well as expanding the acoustic DoFs in terms of a set of $N_{a}$ uncoupled acoustic modes $\Phi_{a}$ (acoustic boundaries considered rigid at the wetted surface). The structural and acoustic expansions become, respectively,

$$
\begin{aligned}
& \mathbf{u}=\sum_{r=1}^{N_{s}} q_{s}\left\{\Phi_{s}\right\}_{r}=\Phi_{s} \mathbf{q}_{s}, \\
& \mathbf{p}=\sum_{r=1}^{N_{a}} q_{a}\left\{\Phi_{a}\right\}_{r}=\Phi_{a} \mathbf{q}_{a},
\end{aligned}
$$

where $\mathbf{q}_{s}$ is the vector of modal amplitudes related to the structural DoFs, $\mathbf{q}_{a}$ is the vector of modal amplitudes related to the acoustic DoFs and $r$ is the index representing the number of the mode.

This procedure yields non-symmetrical coupled modal stiffness and mass matrices. Therefore, obtaining the modal state-space representation of a reduced model derived from CMS can be a difficult task, since it is necessary to invert the coupled modal mass matrix (which is non-diagonal) and the coupling matrix should be fully available.

An alternative to describe a modal state-space for a fully coupled vibro-acoustic system is to apply a variable substitution to the coupled eigenproblem related to Eq. (3) [9]. This procedure is detailed hereafter.

Substituting the component mode expansions in Eqs (7) and (8) into Eq. (3) and pre-multiplying the structural and acoustic parts of the resulting matrix equation, respectively, with the transpose of the structural and acoustic modal vectors yields the undamped modal representation: 


$$
\left(\left[\begin{array}{cc}
\Phi_{s}^{T} \mathbf{K}_{s} \Phi_{s} & \Phi_{s}^{T} \mathbf{K}_{c} \Phi_{a} \\
\mathbf{0} & \Phi_{a}^{T} \mathbf{K}_{a} \Phi_{a}
\end{array}\right]-\omega^{2}\left[\begin{array}{cc}
\Phi_{s}^{T} \mathbf{M}_{s} \Phi_{s} & \mathbf{0} \\
-\rho_{a} \Phi_{a}^{T} \mathbf{K}_{c}^{T} \Phi_{s} & \Phi_{a}^{T} \mathbf{M}_{a} \Phi_{a}
\end{array}\right]\right)\left\{\begin{array}{l}
\mathbf{q}_{s} \\
\mathbf{q}_{a}
\end{array}\right\}=\left\{\begin{array}{l}
\Phi_{s}^{T} \mathbf{F}_{s} \\
\Phi_{a}^{T} \mathbf{F}_{a}
\end{array}\right\}
$$

Since each uncoupled mode is normalized with respect to the uncoupled mass matrices, the homogeneous system of equations related to Eq. (9) can be written as:

$$
\left[\begin{array}{cc}
\Omega_{s}^{2}-\omega^{2} \mathbf{I} & \Phi_{s}^{T} \mathbf{K}_{c} \Phi_{a} \\
\omega^{2} \Phi_{a}^{T} \mathbf{K}_{c}^{T} \Phi_{s}-\rho_{\rho_{a}}\left(\Omega_{a}^{2}-\omega^{2} \mathbf{I}\right)
\end{array}\right]\left\{\begin{array}{l}
\mathbf{q}_{s} \\
\mathbf{q}_{a}
\end{array}\right\}=\left\{\begin{array}{l}
0 \\
0
\end{array}\right\}
$$

where $\Omega_{s}$ and $\Omega_{a}$ are, respectively, the structural and acoustic diagonal matrices of uncoupled natural frequencies.

Equation (10) still results in a non-symmetric eigenproblem and is therefore expensive to solve. The first line of Eq. (10) leads to:

$$
\mathbf{q}_{s}=\omega^{2}\left(\Omega_{s}^{2}\right)^{-1} \mathbf{q}_{s}-\left(\Omega_{s}^{2}\right)^{-1} \Phi_{s}^{T} \mathbf{K}_{c} \Phi_{a} \mathbf{q}_{a}
$$

Applying the substitution $\overline{\mathbf{q}}_{s}=\omega^{2} \mathbf{q}_{s}$ in Eq. (11) yields:

$$
\left\{\begin{array}{l}
\mathbf{q}_{s} \\
\mathbf{q}_{a}
\end{array}\right\}=\left[\begin{array}{cc}
\left(\Omega_{s}^{2}\right)^{-1}-\left(\Omega_{s}^{2}\right)^{-1} \Phi_{s}^{T} \mathbf{K}_{c} \Phi_{a} \\
0 & 1
\end{array}\right]\left\{\begin{array}{l}
\overline{\mathbf{q}}_{s} \\
\mathbf{q}_{a}
\end{array}\right\} .
$$

Using Eq. (12) it is possible to rewrite Eq. (10) as a symmetric system of equations in $\left\{\overline{\mathbf{q}}_{s} \mathbf{q}_{a}\right\}^{T}$ :

$$
\left[\begin{array}{c}
\mathbf{I}-\omega^{2}\left(\Omega_{s}^{2}\right)^{-1} \\
\omega^{2}\left(\Omega_{s}^{2}\right)^{-1} \Phi_{s}^{T} \mathbf{K}_{c} \Phi_{a} \\
\left.\omega^{2}\left(\Omega_{s}^{2}\right)^{-1} \Phi_{a}^{T} \mathbf{K}_{c}^{T} \Phi_{s}-{ }_{\rho_{a}}^{1}\left(\Omega_{a}^{2}-\omega^{2} \mathbf{I}\right)-\omega^{2} \Phi_{a}^{T} \mathbf{K}_{c}^{T} \Phi_{s}^{(} \Omega_{s}^{2}\right)^{-1} \Phi_{s}^{T} \mathbf{K}_{c} \Phi_{a}
\end{array}\right]\left\{\begin{array}{l}
\overline{\mathbf{q}}_{s} \\
\mathbf{q}_{a}
\end{array}\right\}=\left\{\begin{array}{c}
0 \\
0
\end{array}\right\}
$$

The coupled modal vector $\bar{\Phi}$, resulting from the eigenproblem associated with Eq. (13) can be interpreted as the left eigenvector $\Phi_{L}$ of the eigenproblem in Eq. (5). The right eigenvector $\Phi_{R}$ can be retrieved using Eq. (6).

Since the uncoupled bases $\Phi_{a}$ and $\Phi_{s}$ result from symmetric eigenproblems, solving Eq. (13) may seem less demanding when compared to the solution of Eqs (4) and (5). However, the reduction on the computational effort is rather small, as to accurately represent the coupled modes, it is necessary to retain a higher number of uncoupled modes. Nevertheless, the advantage of this method is the possibility of using dedicated software for each component uncoupled modal analysis.

\subsection{Reduced state-space formulation}

Starting from the first order generalized state-space representation:

$$
\begin{aligned}
& \dot{\mathbf{x}}=\mathbf{A} \mathbf{x}+\mathbf{B F}=\left[\begin{array}{cc}
\mathbf{0} & \mathbf{I} \\
-\mathbf{M}^{-1} \mathbf{K}-\mathbf{M}^{-1} \mathbf{C}
\end{array}\right] \mathbf{x}+\left[\begin{array}{c}
\mathbf{0} \\
{[b]}
\end{array}\right] \mathbf{F} \\
& \mathbf{y}=\mathbf{C x}=[[c] \mathbf{0}] \mathbf{x}
\end{aligned}
$$

where $\mathbf{M}, \mathbf{C}$ and $\mathbf{K}$ are respectively the full mass, damping and stiffness matrices, $\mathbf{x}$ is the vector of states, $\mathbf{F}$ is the load vector, $\mathbf{y}$ is the output vector and $[b]$ and $[c]$ are rectangular matrices with ones on the desired DoFs positions and zeros everywhere else.

In this formulation, structural and acoustic DoFs are projected using the modal bases $\Phi_{L}$ and $\Phi_{R}$ and the modal coordinate $\mathbf{q}$ using the following expansion:

$$
\left\{\begin{array}{l}
\mathbf{u} \\
\mathbf{p}
\end{array}\right\}=\sum_{r=1}^{N_{s}+N_{a}} q_{r}\left\{\Phi_{R}\right\}_{r}=\Phi_{R} \mathbf{q}
$$

Moreover, the left and right eigenvectors are normalized such that:

$$
\Phi_{L}^{T}\left[\begin{array}{cc}
\mathbf{K}_{s} & \mathbf{K}_{c} \\
\mathbf{0} & \mathbf{K}_{a}
\end{array}\right] \Phi_{R}=\Omega^{2},
$$




$$
\begin{aligned}
& \Phi_{L}^{T}\left[\begin{array}{cc}
\mathbf{D}_{s} & \mathbf{0} \\
\mathbf{0} & \mathbf{D}_{a}
\end{array}\right] \Phi_{R}=\Gamma, \\
& \Phi_{L}^{T}\left[\begin{array}{cc}
\mathbf{M}_{s} & \mathbf{0} \\
-\rho_{a} \mathbf{K}_{c}^{T} & \mathbf{M}_{a}
\end{array}\right] \Phi_{R}=\mathbf{I},
\end{aligned}
$$

where $\mathbf{I}, \Omega^{2}$ and $\Gamma$ are, respectively, the identity, the squared coupled natural frequencies and the modal damping matrices.

Applying the modal expansion described by Eq. (16) into Eq. (3) and pre-multiplying it by $\Phi_{L}^{T}$, yields:

$$
\Phi_{L}^{T}\left[\begin{array}{cc}
\mathbf{K}_{s} & \mathbf{K}_{c} \\
\mathbf{0} & \mathbf{K}_{a}
\end{array}\right] \Phi_{R} \mathbf{q}+\Phi_{L}^{T}\left[\begin{array}{cc}
\mathbf{D}_{s} & \mathbf{0} \\
\mathbf{0} & \mathbf{D}_{a}
\end{array}\right] \Phi_{R} \dot{\mathbf{q}}+\Phi_{L}^{T}\left[\begin{array}{cc}
\mathbf{M}_{s} & \mathbf{0} \\
-\rho_{a} \mathbf{K}_{c}^{T} & \mathbf{M}_{a}
\end{array}\right] \Phi_{R} \ddot{\mathbf{q}}=\Phi_{L}^{T}\left\{\begin{array}{l}
\mathbf{F}_{s} \\
\mathbf{F}_{a}
\end{array}\right\}
$$

Recalling Eqs (14) and (15), the relations described in Eqs (17), (18) and (19) allow Eq. (20) to be rewritten in a modal state-space form:

$$
\begin{aligned}
& \left\{\begin{array}{c}
\dot{\mathbf{q}} \\
\ddot{\mathbf{q}}
\end{array}\right\}=\left[\begin{array}{cc}
\mathbf{0} & \mathbf{I} \\
-\Omega^{2} & -\Gamma
\end{array}\right]\left\{\begin{array}{c}
\mathbf{q} \\
\dot{\mathbf{q}}
\end{array}\right\}+\left[\begin{array}{c}
\mathbf{0} \\
\Phi_{L}^{T}[b]
\end{array}\right]\left\{\begin{array}{l}
\mathbf{F}_{s} \\
\mathbf{F}_{a}
\end{array}\right\} \\
& \mathbf{y}=\left[[c] \Phi_{R}^{T} \mathbf{0}\right]\left\{\begin{array}{c}
\mathbf{q} \\
\dot{\mathbf{q}}
\end{array}\right\}
\end{aligned}
$$

In this way, a fully coupled vibro-acoustic system can be written in a reduced modal state-space formulation with order $(2 N \times 2 N)$. Since the coupled vibro-acoustic model is derived from uncoupled structural and acoustic modal bases, it is necessary to retain a higher number of modes in order to accurately represent the dynamic behavior in the desired frequency range. Therefore, both uncoupled modal bases were evaluated up to $400 \mathrm{~Hz}$, which is adequate to represent the vibro-acoustic system in the frequency range of interest ( 0 to $200 \mathrm{~Hz}$ ). Applying the aforementioned procedure, the original 24192 DoFs (23196 unconstrained acoustic and 1026 unconstrained structural) have been reduced to a SS model with 214 states related to the 107 coupled modes in the 0 to $400 \mathrm{~Hz}$ frequency range. The inputs can comprise forces applied to the firewall and volume velocity sources in either the cavities. The outputs are structural displacement and acoustic pressure.

\subsection{SAP model}

In active control, sensors, actuators and structure are put together in such a way that the level of interaction between those elements turns any separately approach of the subsystems impossible [10]. Therefore, a unifying approach that takes into account the fully coupled system is needed. Among the present solutions are those based on the inclusion of sensor, actuators and control laws models into CAE software such as FE, multibody systems (MBS), etc. [11]. Another possible solution, when two time domain simulations are involved, is the co-simulation, usually applied to MBS models and a controller in Simulink [12]. Finally, the methodology adopted in this work involves the inclusion of a reduced model of the vibro-acoustic systems into the control system design environment (Matlab/Simulink), where the interaction between structure and sensor/actuator is eventually taken into account.

A set of collocated SAPs is placed on the firewall to realize the collocated velocity feedback in order to reduce the noise transmitted from the EC to the PC (Fig. 5a). Collocated accelerometers and inertial shakers are used as structural SAPs. Appropriate accelerometers are selected, for the frequency range of interest, such that the voltage signal generated by these devices can be considered proportional to the measured quantity. However, for the inertial shakers, a more detailed model for the electro-mechanical coupling within the actuator and its interaction with the structure is considered.

The causes and effects of the interaction between electrodynamic exciters and the structure under test (SUT) has been an issue for the experimentalists since the very beginning of modal analysis as in $[13,14]$ and still is a subject of research [15-18]. Figure 6 shows the electro-mechanical model of an inertial shaker. Equations (23) and (24) describe the dynamics of the coupled electro-mechanical system with the amplifier set in voltage mode of operation. As it can be seen, the coupling occurs in Eq. (23) as the right-hand side expresses the magnetic force (acting on the moving mass $m_{i}$ ) proportionally to the current $I$ flowing on the circuit; and in Eq. (24) in which the electric potential 


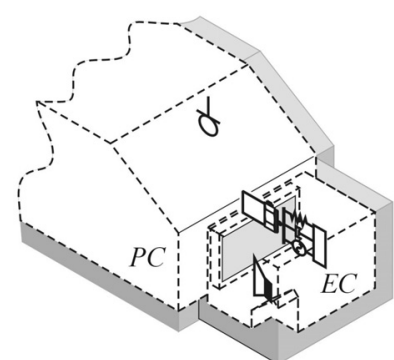

(a)

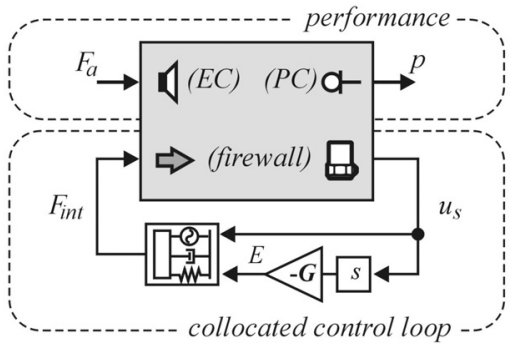

(b)

Fig. 5. Control scheme (a) positions of sensors and actuators and (b) block diagram.

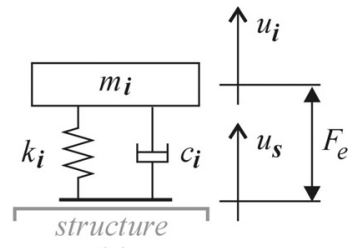

(a)

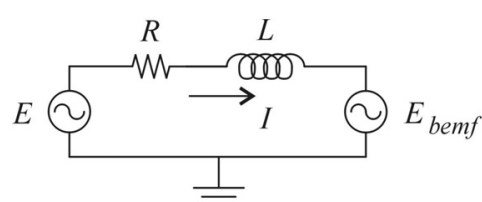

(b)

Fig. 6. Electro-mechanical model of an inertial shaker: (a) mechanical and (b) electrical model.

$k f\left(\dot{u}_{i}-\dot{u}_{s}\right)=E_{\text {bem } f}$ is the voltage generate by the movement of coil in the magnetic field, thus written in terms of the relative velocity between the SUT driving point and the shaker moving mass. More detailed information about the shaker dynamics as well as the amplifier modes of operation can be found in $[16,17]$.

$$
\begin{aligned}
& m_{i} \ddot{u}_{i}+d_{i}\left(\dot{u}_{i}-\dot{u}_{s}\right)+k_{i}\left(u_{i}-u_{s}\right)=k f I \\
& R I+L \dot{I}+k f\left(\dot{u}_{i}-\dot{u}_{s}\right)=E
\end{aligned}
$$

As mentioned before, the objective of the proposed modeling procedure is to include the actuator (considering its interaction with the SUT) in the controller design environment. At this point, the SUT is represented by a reduced modal model, from which the displacement of the driving point is available. Since the shaker is rigidly connected to the structure, the movement of the base will be the same as the SUT driving point. In this way the inertial shaker can be represented by the moving mass and the active interface (passive suspension plus electromagnetic force). As proposed by [19] for a hybrid isolation mount, the shaker active interface can also be modeled as a lumped impedance element that contains the passive and active parameters (Fig. 7). Thus, it is possible to include one or more inertial shakers, given the driving point displacements and the driving voltage $E$.

It is important to state that the feedback gain(s) for the structural SAP(s) should be optimized with respect to the pressure at the driver's ear, rather than the firewall vibration. This ASAC strategy is applicable when the acoustic source is transmitted into a cavity through a limited number of structural paths [20]. As shown in Fig. 5(b), in the adopted ASAC strategy, the structural sensors and actuators are involved in the control loop whereas the performance is evaluated at the acoustic sensor.

\section{Results}

The next subsections show the simulation results obtained using the proposed methodology. Subsection 3.1 deals with the passive system simulation and the interaction between the firewall and the exciter, while 3.2 shows how the modeling procedure can be applied to ASAC simulations where one or more SAPs are used in velocity feedback controllers. 


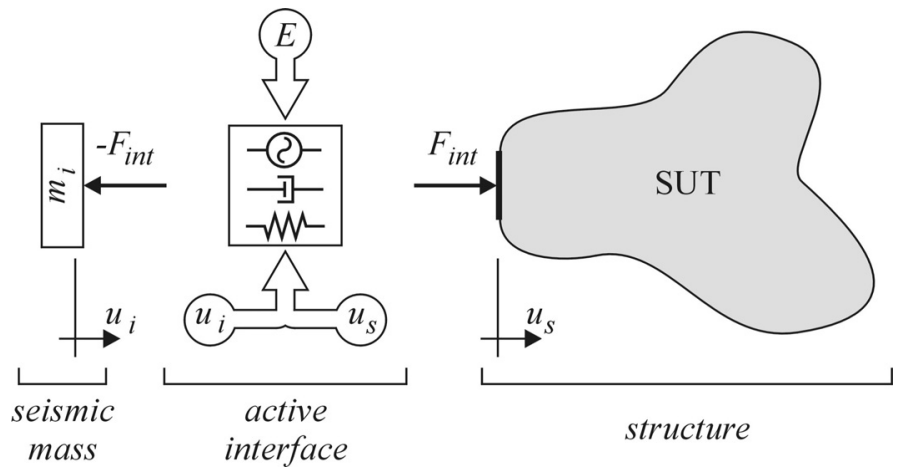

Fig. 7. Inertial shaker active interface model.

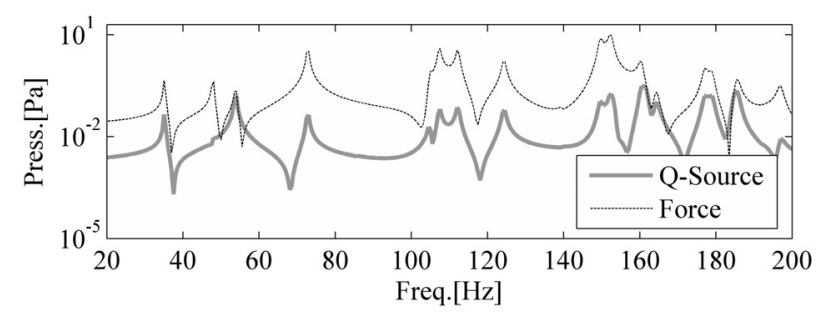

(a)

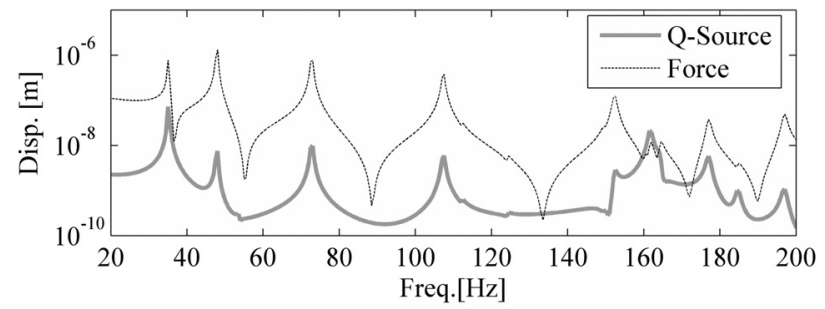

(b)

Fig. 8. State-space forced responses: (a) acoustic output and (b) structural output.

\subsection{Passive results}

A 2-input/2-output state-space model has been built (Fig. 5b). The structural and acoustical inputs are respectively, the force $\left(F_{\text {int }}\right)$ and the volume velocity $\left(F_{a}\right)$; the outputs are the collocated displacement $\left(u_{s}\right)$ on the firewall driving point and pressure $(p)$ at the driver's head position.

Figure 8 illustrates the forced responses calculated with the state-space model for both kinds of inputs: volumevelocity $\left(1 \times 10^{-6} \mathrm{~m}^{3} / \mathrm{s}\right)$ from the acoustic source in the EC and normal force $(1 \mathrm{~N})$ at an arbitrary position on the firewall. The graphics show the pressure at the driver's head position and the displacement from the driving point on the firewall. From these graphs, it can be seen that the model is indeed coupled.

The forced responses on Fig. 8 came merely from the system transfer functions, i.e., the input force is assumed ideal. However, if the model of the exciter is included in the simulation, it is possible to observe phenomena inherent to the use of such electrodynamic devices, e.g. force drop-off. Figure 9 shows, in the upper part, the structural FRF and in the lower part, a comparison of the idealized force input and the actual load provided by an inertial shaker. It can be seen that in the low frequency range and in the vicinities of structural resonances, the force level drops, as a result of shaker/structure interaction $[13,16]$.

The mass loading and drops in the exaltation force can lead to errors in the experimental FRFs [17,18] but mainly, as far as the active control system is concerned, can result in overestimated authority and performance. 


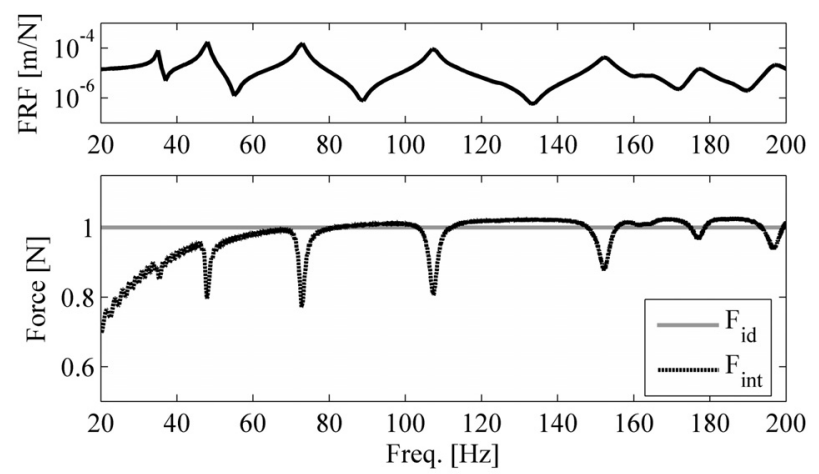

Fig. 9. System driving point FRF and input forces.

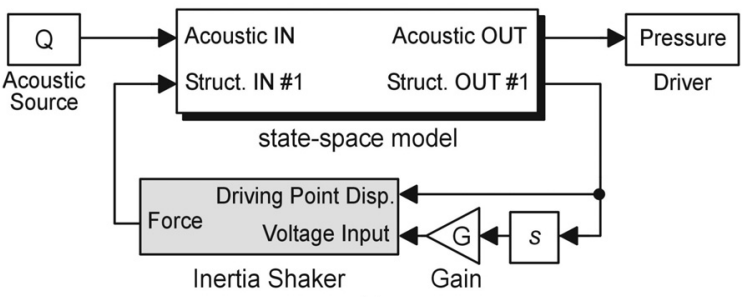

(a)

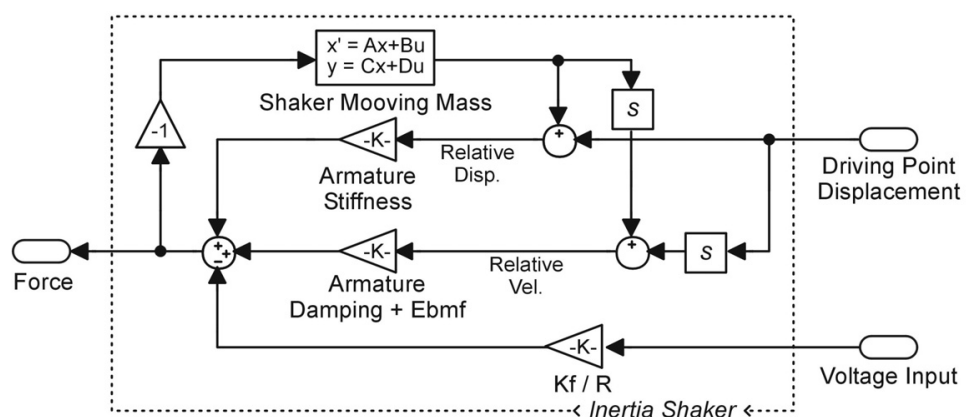

(b)

Fig. 10. Block diagrams: (a) ASAC simulation (b) detailed shaker block.

\subsection{ASAC simulation}

The choice of using only structural sensors and actuators in this ASAC approach is based on the robustness of the control system. Since feedback is going to be used, an important aspect to the efficiency and stability of the control system is the transfer function between the sensor and actuator. The phase angle between these two signals should be bounded by $+/-90^{\circ}$, otherwise the system can become unstable. Usually, the use of acoustic sensors and/or actuators presents a fast phase loss, which would impose severe limitations to the controller frequency range [20]. However, since the SAP is collocated it can be proved that the control system acts in fact like a passive system and stability is always guaranteed, independent of the feedback gain [10,21].

Also, the structural transfer functions are much less sensitive to typical changes in this kind of systems, such as open window or the placement of more people inside the vehicle. On the other hand, the control system just senses (directly) the structural DoFs. As a result, it is expected that only the predominantly structural resonances will be affected by the controller, while the predominantly acoustic ones may not be. 


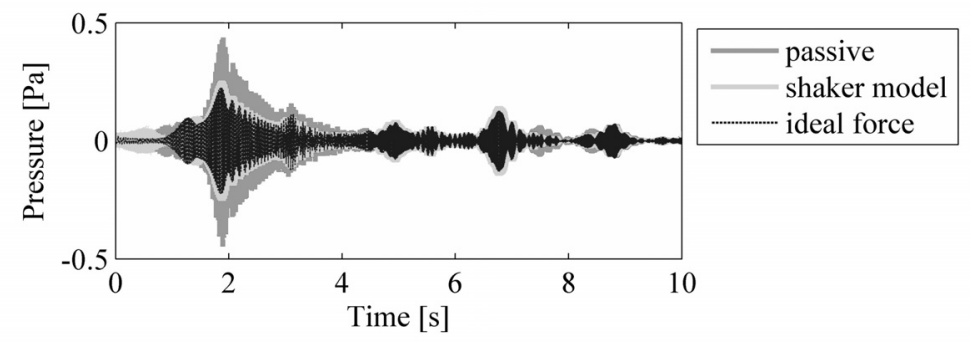

Fig. 11. Pressure at the drive's head position for different conditions $(\mathrm{G}=450 \mathrm{~V} /(\mathrm{m} / \mathrm{s}))$.

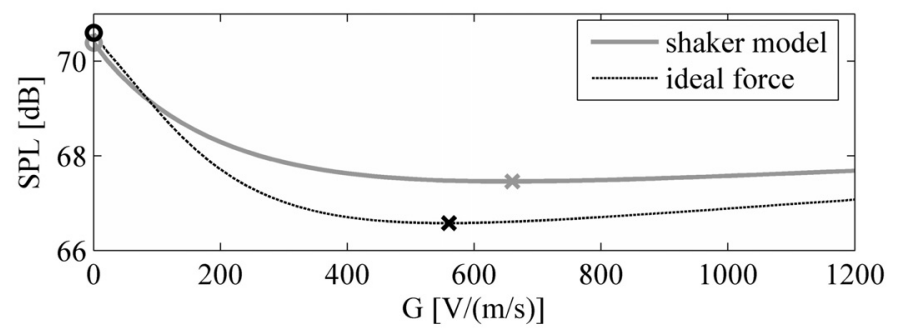

Fig. 12. Sound pressure level at driver's head position: (o) passive and (x) optimal active.

The description of the vibro-acoustic system as a state-space modal model allows closed-loop simulations in time and frequency domain. The collocated velocity feedback control loop can be implemented in Matlab/Simulink (Fig. 10a).

The basic principle of this controller is to feed the actuator with an amplified voltage proportional to the velocity from its collocated sensor. Therefore, the inertial shaker block is connected to the structural input/output ports of the state-space model in a velocity feedback configuration. Figure 10(b) shows in more detail the inertial shaker block diagram. The structure driving point and the shaker moving mass displacements (and velocities) are used, together with the input voltage, to compute the interface force $\left(F_{\text {int }}\right)$ acting on the system.

Equation (25) is the differential equation governing the block diagram in Fig. 10(b). It is based on Eqs (23) and (24) where the coil inductance is neglected as suggested by [22,23]. Since the system is in a velocity feedback configuration, the input voltage $E$ will be proportional to the driving point velocity (Fig. 10a).

$$
m_{i} \ddot{u}_{i}+\left(d_{i}+\begin{array}{c}
k f^{2} \\
R
\end{array}\right)\left(\dot{u}_{i}-\dot{u}_{s}\right)+k_{i}\left(u_{i}-u_{s}\right)={ }_{R}^{k f} E
$$

Figure 11 shows the pressure at the driver's head position for a chirp acoustic disturbance and different conditions: passive system, active system with idealized velocity feedback and active system with shaker model (as in Fig. 10). As expected, it is possible to notice that the idealized force approach overestimate the control performance, mainly in the low frequency range and close to resonances. As a result, for the same feedback gain $(\mathrm{G}=450 \mathrm{~V} /(\mathrm{m} / \mathrm{s}))$, the estimated reduction on the sound pressure level (SPL) for the idealized force approach is $4 \mathrm{~dB}$ while, when the shaker model is included, it is $2.8 \mathrm{~dB}$.

Figure 12 shows the overall noise reduction as a function of the feedback gain for both, idealized force and shaker model approaches. As it can be seen, in either case there is an optimum gain with respect to the SPL at the drive's head position. The performance evaluated with idealized forces is almost ever overestimated when compared to the one calculated with the shaker model. The latter is just not true for low values of G, where the inclusion of the shaker model results in slightly lower SPL. This effect can be noticed even for $\mathrm{G}=0$, i.e. the passive system, as the shaker represents an additional mass-spring-damper system attached to the firewall.

It is important to notice that the positioning of the SAP plays an important role in the maximum achievable noise reduction. One could also consider the firewall thickness as a variable in this design process, resulting in a multi-disciplinary optimization task. The use of state-space models, as the one described in this paper, for the evaluation and optimization of such active control systems can be seen in more details in [24,25]. 


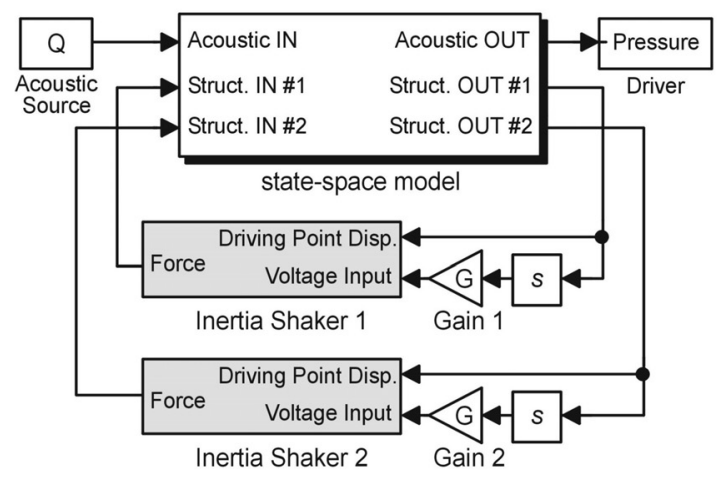

(a)

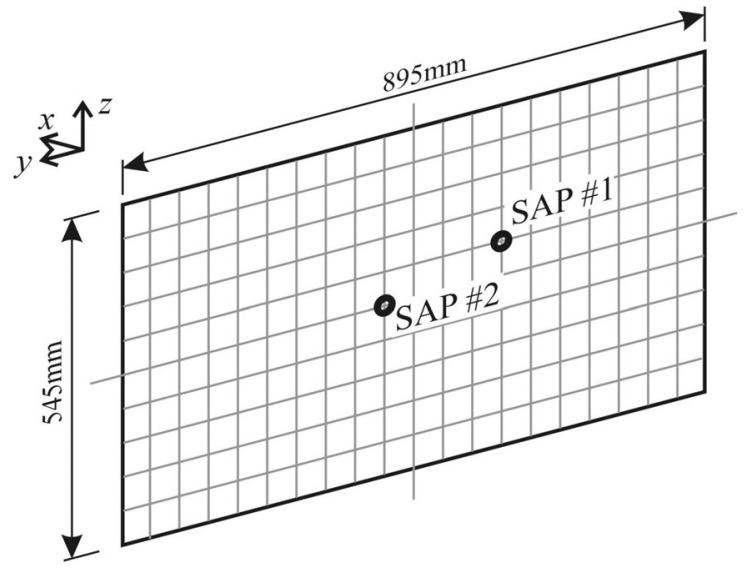

(b)

Fig. 13. 2 SAPs ASAC: (a) block diagram and (b) SAP positions on the firewall.

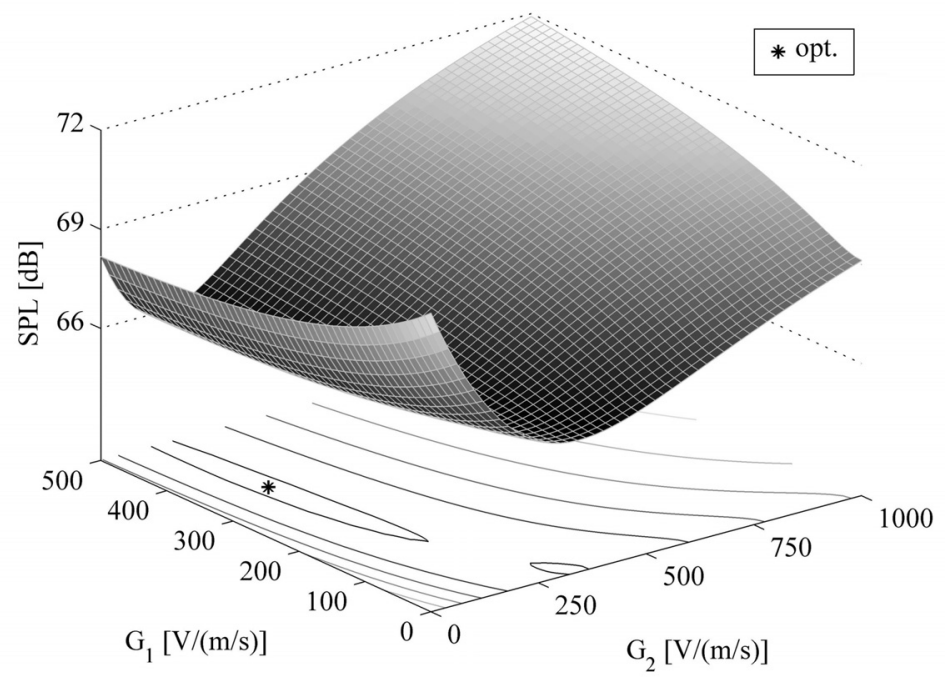

Fig. 14. Solution surface for 2 SAPs: Performance X feedback gains.

Furthermore, the 2.8 dB SPL reduction achieved with one arbitrarily placed SAP can be improved if more SAPs are used. To demonstrate a simulation scheme with multiple SAPs, a configuration with 2 collocated SAPs in a decentralized velocity-feedback control loop is applied to the firewall (Fig. 13). Again, as an optimization of the SAPs location is out of the scope of this work, their placement is made upon previous experience. As demonstrated by [26], an arbitrary placement can lead to satisfactory results if the feedback gains are optimized. The state-space model has been augmented with the extra structural input/output point (Fig. 13a), resulting in the 2 SAPs positions depicted in Fig. 13(b).

Figure 14 shows how the control system performance varies with respect to the feedback gains $G_{1}$ and $G_{2}$. The performance is considered as the SPL reduction in $\mathrm{dB}$ at the driver's ear. It is possible to access the optimal gains for both SAPs based on the solution surface on Fig. 14. As a result, the overall system performance is $5.1 \mathrm{~dB}$, with feedback gains $G_{1}$ and $G_{2}$ respectively -350 and $-160 \mathrm{~V} /(\mathrm{m} / \mathrm{s})$. 


\section{Summary and conclusions}

A modeling procedure for ASAC simulation, considering a fully coupled vibro-acoustic system has been presented. Structural FE models are used to calculate a vibro-acoustic coupled modal base, which is eventually exported and formulated as a state-space modal model. Finally, the models for the inertial shakers are incorporated and the control system can be implemented.

The displacement/pressure Eulerian modal base allows the representation of the vibro-acoustic FE model in a state-space formulation featuring coupled structural and acoustical inputs and outputs.

The inertial shaker was modeled as a lumped mass connected by an active interface to the structure. The results obtained through this time-domain procedure, as the force drop-off phenomenon, are similar to those found in the literature for electrodynamic shakers. Hence, the modeling procedure succeeded in representing such a coupled electro-vibro-acoustic system. The overestimate error from the idealized force controller reached up to $1.2 \mathrm{~dB}$ in $2.8 \mathrm{~dB}$ overall reduction, highlighting the importance of including the SAP dynamics.

The ASAC simulation allows the inclusion of any kind of controller that uses structural or acoustical sensors and actuators. As an example, decentralized velocity feedback with 1 and 2 SAPs were presented. A Total reduction of $5.1 \mathrm{~dB}$ was achieved with 2 SAPs. It is probable that the SAP placement is not optimum, therefore an increase in the achievable reduction can still be reached, even if the current configuration ( 2 collocated SAPs) is kept. This solution could be accessed by an optimization routine that takes into account not only the feedback gain, but also the SAP placement. The amount of reduction could also be increased if more SAPs are placed simultaneously on the firewall. However, the design space grows exponentially with respect to the number of variables, which significantly increases the computational effort for the optimization procedure. The use of reduced models, as shown in this paper, is crucial for the feasibility of such optimizations.

Besides all the advantages of a collocated velocity-feedback controller, and the fact that structural SAPs would be robust against changes on the acoustic system, it presented a rather weak effect on damping the predominantly acoustic modes. However, as far as the primary source has a random nature, e.g. aerodynamic or tire noise, the feedback approach is probably the most convenient solution. If the objective is to prevent the transmission of engine noise to the passenger compartment, and considering that a good reference signal is available (engine speed), a feedforward approach may lead to better results. In any case, the modeling approach proposed here fulfils the simulation requirements, providing a more accurate model of the plant which can include sensors and actuators dynamics.

\section{Acknowledgments}

The research of Leopoldo P.R. de Oliveira is financed by a scholarship in the framework of a selective bilateral agreement between the KU Leuven and the University of São Paulo. Part of this research was done in the framework of the European FP6 Integrated Project: Intelligent Materials for Active Noise Reduction - InMAR.

\section{References}

[1] H. Van der Auweraer, K. Janssens, L.P.R. Oliveira, M.M. Silva and W. Desmet, Virtual Prototyping for Sound Quality Design of Automobiles, S V Sound and Vibration 41(4), (2007), 26-30.

[2] J.I. Mohammed and S.J. Elliott, Active control of fully coupled structural-acoustic system, Proceeding of Inter-Noise 2005, Rio de JaneiroBrazil, 2005, 10

[3] P. Sas, C. Bao, F. Augusztinovicz and W. Desmet, Active control of sound transmission through a double panel partition, Journal of Sound and Vibration 180(4) (1995), 609-625.

[4] W. Desmet, B. Pluymers and P. Sas, Vibro-acoustic analysis procedures for the evaluation of the sound insulation characteristics of agricultural machinery cabins, Journal of Sound and Vibration 266(3) (2003), 407-441.

[5] J.P. Coyette and Y. Dubois-Pèlerin, An Efficient Coupling Procedure for Handling Large Size Interior Structural-Acoustic Problems, Proceedings of ISMA-19, Leuven - Belgium, 1994, pp. 729-738.

[6] N. El-Masri, M. Tournour and C. McCulloch, Meshing procedure for Vibro-Acoustic Models, Proceedings of ISMA 2002, Leuven Belgium, 2002, pp. 2151-2157. 
[7] W. Desmet and D. Vandepite, Finite Element Method in Acoustics, ISAAC 15 - Seminar on Advanced Techniques in Applied and Numerical Acoustics, Leuven - Belgium, 2004, 48.

[8] J. Luo and H.C. Gea, Modal Sensitivity analysis of coupled acoustic-structural systems, Journal of Vibration and Acoustics 119 (1997), 545-550.

[9] Sysnoise rev. 5.5 User's Manual, LMS International, Leuven, Belgium, 2000.

[10] A. Preumont, Vibration Control of Active Structures: An Introduction, Kluwer Academic Publishers, 2002 , Ed.2.

[11] P. Fisette, O. Brüls and J. Swevers, Multiphysics Modeling of Mechatronic Multibody Systems, Proceedings of the International Conference on Noise and Vibration Engineering - ISMA 2006, Leuven - Belgium, 2006, pp. 41-68.

[12] M.M. da Silva, W. Desmet and H. Van Brussel, Design of mechatronic systems with configuration-dependent dynamics: simulation and optimization, IEEE/ASME Trans. on Mechatronics 13(6) (2008), 638-646.

[13] G.R. Tomlinson, Force Distortion in Resonance Testing of Structures with Electrodynamic Vibration Exciters, Journal of Sound and Vibration 63(3) (1979), 337-350.

[14] K. Unholtz, Vibration testing machines - Shok and Vibration Handbook, McGraw-Hill Book Co., New York, 1961, v.2, pp. 25.1-25.74, Ed.1.

[15] G.F. Lang, Understanding the Physics of Electrodynamic Shaker Performance, Sound and Vibration - October 2001, pp. 1-9.

[16] K.G. McConnell, Vibration Testing: Theory and Practice, John Wiley \& Sons, 1995, NY - USA, Ed.1.

[17] P.S. Varoto and L.P.R. Oliveira, On the Force Drop-off Phenomenon in Shaker Testing in Experimental Modal Analysis, Shock and Vibration 9 (4-5SPEC.) (2002), 165-175.

[18] P.S. Varoto and L.P.R. Oliveira, Interaction between a vibration exciter and the structure under test, $S$ V Sound and Vibration 36(10), (2002), 20-26.

[19] S. Herold, H. Atzrodt, D. Mayer and M. Thomaier, Integration of Different Approaches to Simulate Active Structures for Automotive Applications, Proceedings of Forum Acusticum 2005, Budapest - Hungary, 2005, pp. 909-914.

[20] P.A. Nelson and S.J. Elliott, Active Control of Sound, Academic Press, 1992, Ed.1.

[21] K. Henrioulle and P. Sas, Experimental validation of a collocated PVDF volume velocity sensor/actuator pair, Journal of Sound and Vibration 265(3) (2003), 489-506.

[22] D.K. Rao, Electrodynamic Interaction Between a Resonating Structure and an Exciter, Proceedings of the 5th International Modal Analysis Conference - V IMAC, 1987, v.2, pp. 1142-1150.

[23] N.M.M. Maia and J.M.M. Silva, Theoretical and Experimental Modal Analisys, Research Studies Pess Ltd., 1997, England, Ed.1.

[24] L.P.R. de Oliveira, M.M. da Silva, P. Sas, H. Van Brussel and W. Desmet, Concurrent mechatronic design approach for active control of cavity noise, Journal of Sound and Vibration 314 (2008), 507-525.

[25] L.P.R. de Oliveira, K. Janssens, P. Gajdatsy, H. Van der Auweraer, P.S. Varoto, P. Sas and W. Desmet, Active sound quality control of engine induced cavity noise, Mechanical Systems and Signal Processing 23 (2009), 476-488.

[26] L.P.R. Oliveira, B. Stallaert, W. Desmet, J. Swevers and P. Sas, Optimisation Strategies for Decentralized ASAC, Proceeding of Forum Acusticum 2005, 29 Aug. - 2 Sep 2005, Budapest, pp. 875-880. 

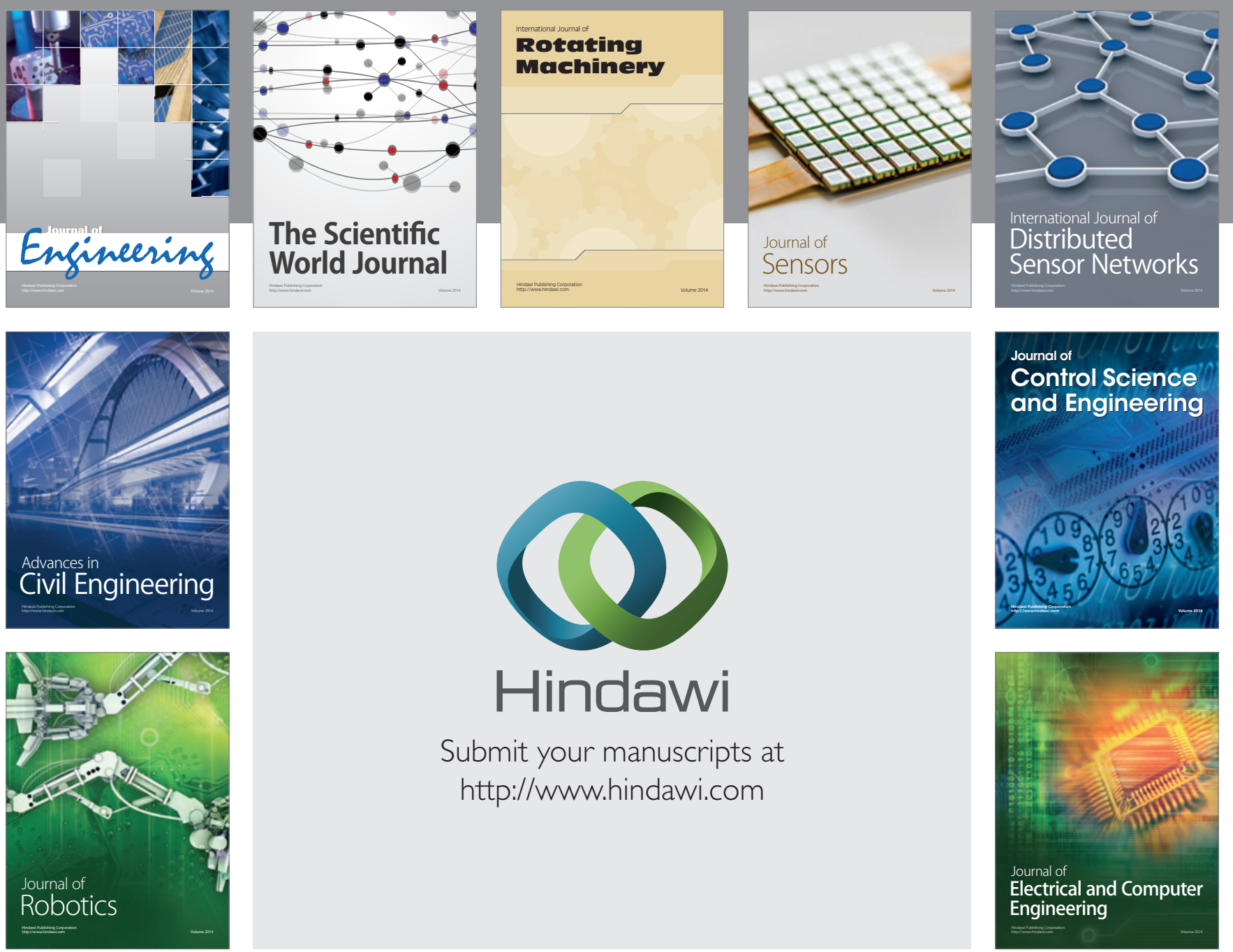

Submit your manuscripts at

http://www.hindawi.com
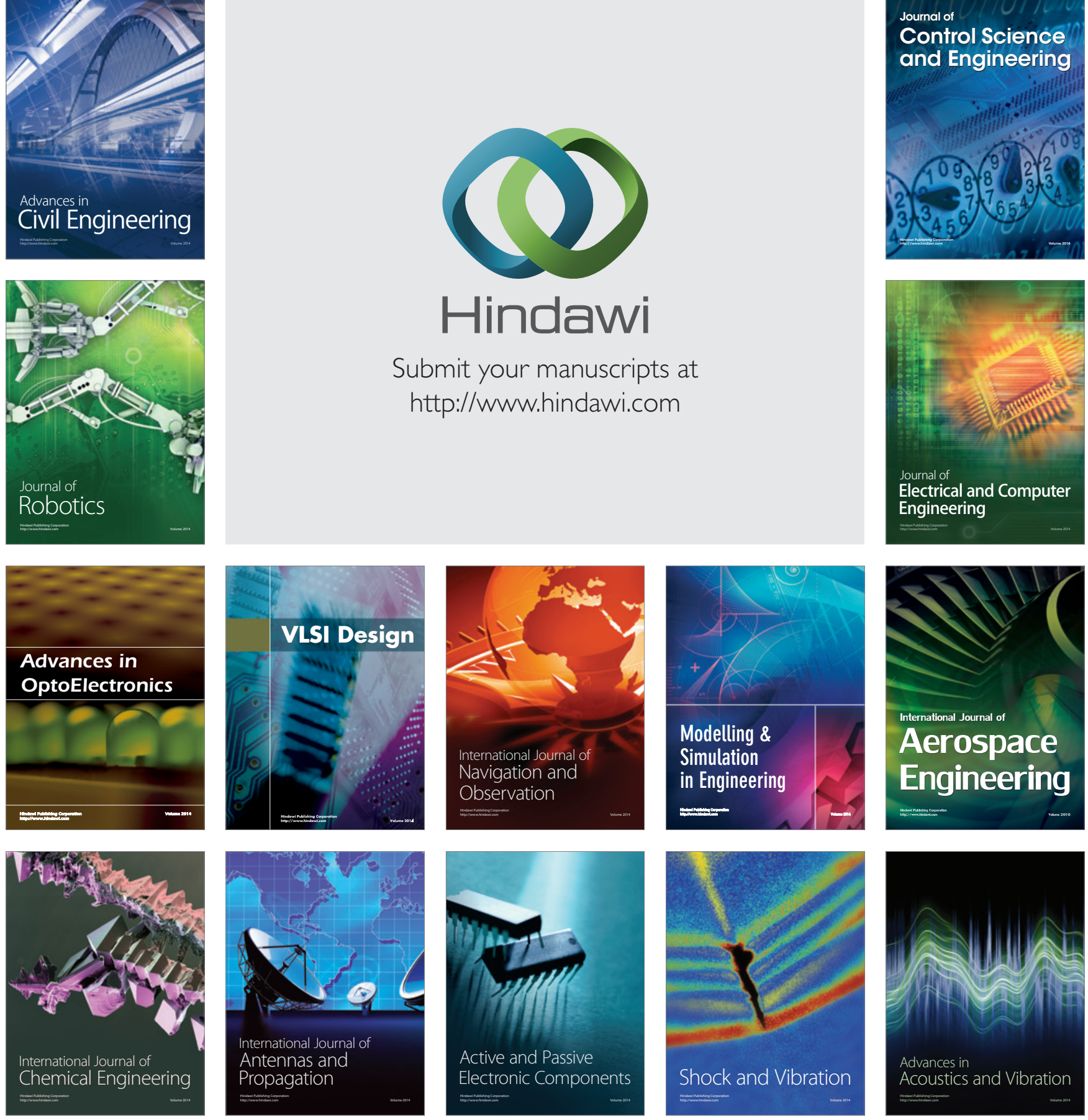